\title{
L'artisanat rural dans la Beauce au XIXe siècle
} Jean-Claude Farcy

\section{Citer ce document / Cite this document :}

Farcy Jean-Claude. L'artisanat rural dans la Beauce au XIXe siècle. In: Histoire, économie et société, 1986, $5^{\mathrm{e}}$ année, $\mathrm{n}^{\circ} 4 . \mathrm{pp}^{\mathrm{a}}$ 573-590;

doi : 10.3406/hes.1986.2349

http://www.persee.fr/doc/hes_0752-5702_1986_num_5_4_2349

Document généré le 15/06/2016 


\title{
Résumé
}

Résumé Dans une région agricole riche comme la Beauce, l'artisanat et le commerce occupent une place importante au sein de la société rurale. L'analyse des recensements et des archives financières montre que cette petite bourgeoisie rurale, qui fait travailler un actif sur quatre au XIXe siècle, est proche, par la possession d'un capital, par le travail de la terre comme par ses niveaux de fortune du milieu des petits cultivateurs. Mais cette catégorie sociale est aussi très hétérogène : l'activité commerciale, très minoritaire, mais en progrès au cours du XIXe siècle, peut accéder à la bourgeoisie « moyenne » alors que la majorité des artisans a une condition plus modeste, parfois voisine de celle des salariés agricoles. L'importance numérique du groupe comme sa position sociale intermédiaire contribuent à atténuer les contrastes sociaux dans une campagne dominée par le capitalisme agraire.

\begin{abstract}
Abstract In a rich agricultural region like the Beauce, the handicraftsman and the merchant occupy an important place in the heart of the rural society. An analysis of census returns and financial archives shows that this petit rural bourgeoisie, which was responsible for the employment of one out of four active workers in the nineteenth century, is very close, by virtue of its possession of capital, by the work on the land, as well as by the levai of its wealth, to the world of the small farmer. But this social category is also very heterogeneous : commercial activity, very much in the minority, but increasing in the course of the nineteenth century, could reach the level of middle bourgeoisie, whereas the majority of artisans have a more modest condition, sometimes bordering on the level of agricultural wageearners. The numerical importance of the group Wee its intermediary social position contributes to attenuate the social contrasts in a countryside dominated by agrarian capitalism.
\end{abstract}




\title{
L'ARTISANAT RURAL DANS LA BEAUCE AU XIXe SIECLE
}

\author{
par Jean-Claude FARCY
}

\section{Résumé}

Dans une région agricole riche comme la Beauce, l'artisanat et le commerce occupent une place importante au sein de la société rurale. L'analyse des recensements et des archives financières montre que cette petite bourgeoisie rurale, qui fait travailler un actif sur quatre au XIXe siècle, est proche, par la possession d'un capital, par le travail de la terre comme par ses niveaux de fortune du milieu des petits cultivateurs. Mais cette catégorie sociale est aussi très hétérogène : l'activité commerciale, très minoritaire, mais en progrès au cours du XIXe siècle, peut accéder à la bourgeoisie " moyenne " alors que la majorité des artisans a une condition plus modeste, parfois voisine de celle des salariés agricoles. L'importance numérique du groupe comme sa position sociale intermédiaire contribuent à atténuer les contrastes sociaux dans une campagne dominée par le capitalisme agraire.

\begin{abstract}
In a rich agricultural region like the Beauce, the handicraftsman and the merchant occupy an important place in the heart of the rural society. An analysis of census returns and financial archives shows that this petit rural bourgeoisie, which was responsible for the employment of one out of four active workers in the nineteenth century, is very close, by virtue of its possession of capital, by the work on the land, as well as by the leval of its wealth, to the world of the small farmer. But this social category is also very heterogeneous : commercial activity, very much in the minority, but increasing in the course of the nineteenth century, could reach the level of middle bourgeoisie, whereas the majority of artisans have a more modest condition, sometimes bordering on the level of agricultural wage earners. The numerical importance of the group like its intermediary social position contributes to attenuate the social contrasts in a countryside dominated by agrarian capitalism.
\end{abstract}

La partie la plus méconnue de la petite bourgeoisie est sans doute celle de l'artisanat et du petit commerce dans les campagnes. Cette méconnaissance se comprend dans la mesure où il est plus significatif d'étudier la petite bourgeoisie confrontée à l'essor du capitalisme industriel et commercial, et cette étude trouve son lieu naturel dans les zones urbaines.

Pourtant, la place de l'artisanat et de la boutique est importante dans les régions rurales, et dans la Beauce d'Eure-et-Loir, région purement agricole, sans aucune activité industrielle au XIXe siècle, c'est autour du quart de la population active qui relève de ce secteur économique au milieu du XIXe siècle.

Le manque d'homogénéité de cette couche sociale rend son étude difficile. La diversité se retrouve aussi bien pour la situation par rapport aux moyens de production, la nature de l'activité, les métiers exercés, que pour la richesse et la place dans la société. A suivre les descriptions des fonctionnaires du XIXe siècle, l'artisanat et le commerce regroupent, en Beauce, tous ceux qui ne travaillent pas directement la terre, si l'on excepte quelques dizaines de membres des professions libérales par canton. C'est de cette "définition 》 large que nous partirons pour mettre en cuvre les différentes sources d'archives dont nous disposons. Ce choix quant à la délimitation du groupe social étudié, recoupe pour une grande part la réalité, et nous pouvons faire nôtre la remarque d'E. Labrousse : 
« L'artisanat des sociétés pré-industrielles couvre un immense front économique. Il assume la masse de la production et de la distribution des secteurs secondaires et tertiaires. A ses échelons infimes et sous ses formes les plus frustres, économiquement il touche au salariat, il est un quasi-salariat. A ses échelons les plus élevés, économiquement, il touche à la petite bourgeoisie commerçante, il est une quasi-bourgeoisie, relevant du monde de la petite entreprise. \(1)

Si l'on suit également le conseil du même auteur de ne pas séparer l'étude de l'artisanat de celle du petit commerce, la délimitation fondée essentiellement sur le genre d'activité est dans un premier temps valable. Elle permet du moins de situer l'importance numérique et l'évolution du groupe social concerné, étant entendu que l'analyse des différenciations internes du groupe doit être faite, en cherchant à distinguer ce qui relève de la bourgeoisie ou ce qui se rapproche plus du prolétariat pour reprendre les frontières sociales suggérées par E. Labrousse.

C'est en mettant l'accent sur les structures internes de cet artisanat rural que nous pourrons le mieux répondre aux interrogations récentes sur la petite bourgeoisie " classe méconnue »(2). Pour ce faire nous utiliserons principalement la documentation d'archives propre au milieu artisanal, c'est-à-dire la statistique des patentés. A défaut des matrices de patentes, inexistantes aux Archives départementales d'Eureet-Loir (3), on se contentera des Etats des montants des rôles généraux des contributions (4) qui permettent le relevé du nombre des patentés par année. Nous retiendrons surtout les Registres de renseignements statistiques et administratifs des contrôles des contributions directes, qui, tous les trois ans, de 1846 à 1879, nous donnent, à l'échelon communal, un classement des différents métiers, en particulier selon l'importance de l'établissement commercial ou artisanal. Ces registres sont donc la source de base pour l'étude de la hiérarchie interne de la petite bourgeoisie artisanale. Toutefois les modifications de la législation dans le domaine des patentes rendent cette source moins fiable pour la deuxième moitié du XIXe siècle et il faut confronter les indications qu'elle fournit avec d'autres sources telles que les listes de conscrits (5), les listes nominatives des recensements de population (6) ou les archives d'une autre administration financière, celle de l'Enregistrement (7). Cette confrontation des sources permet de mieux cerner l'importance et l'évolution numérique de cet artisanat rural, ses structures et sa hiérarchie interne, et sa place dans la société rurale.

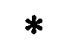

* * *

Au milieu du XIXe siècle, si l'on considère la partie beauceronne de l'Eure-etLoir (8) on constate que les professions artisanales et commerciales ne sont pas loin de représenter le quart de la population active. Dans un échantillon des jeunes relevé sur les listes de conscrits pour les années 1852,1853 et 1854 , nous en avons 607 sur 2469 , soit $24,58 \%$ qui relèvent de ces professions. Un jeune sur quatre, dans la campagne beauceronne, travaille dans ce secteur. Est-ce à l'image de la société rurale dans son ensemble?

Les récapitulatifs communaux du recensement de population effectué en 1851 nous apportent une vérification puisqu'ils indiquent le nombre de "patrons » de 
l'artisanat et du commerce ainsi que le nombre d'ouvriers employés. Pour le même cadre géographique, nous avons $23,64 \%$ des actifs masculins qui font partie de ce secteur social (9) dont 5794 «patrons » et 3115 « salariés », soit un peu plus d'un salarié pour deux patrons, ce qui témoigne d'une très faible concentration économique.

La statistique des patentés apporte des données concordantes. Il y a, toujours pour l'ensemble de la partie purement rurale de la Beauce d'Eure-et-Loir, 5717 patentés d'après le Montant des rôles des contributions de 1851 , alors que nous avons relevé 5794 artisans et commerçants « à leur compte » dans le recensement de 1851 . $\mathrm{Au}$ milieu du XIXe siècle, la liste des patentés donne donc une image correcte pour situer la place du groupe des artisans et commerçants dans l'ensemble de la société. Dans la Beauce, c'est près d'un actif sur quatre qui travaille dans ce secteur. Si l'on ne tient pas compte des salariés, c'est entre 15 et $17 \%$ de la population active qui appartient à ce groupe social. Cette place importante est à mettre en rapport avec une certaine autonomie de la Beauce sur le plan économique. Etant donné la faible modernisation des techniques agricoles, une foule d'artisans contribue à fabriquer et réparer l'outillage agricole, et à transformer les productions agricoles, animant ainsi un important secteur alimentaire. L'analyse interne de cet artisanat rural nous permettra de préciser davantage les raisons de ce rôle important de l'artisanat dans une région rurale.

Ce rôle ne semble pas se modifier réellement tout au long du XIXe siècle. Certes il est difficile de mesurer l'évolution numérique de l'artisanat et du commerce. Si l'on considère d'abord les enseignements de la statistique des patentés, d'après les Montants des rôles des contributions, le nombre de ceux-ci passe de 5717 en 1851 à 4713 en 1911 , soit une diminution de $17,5 \%$. Mais comme durant la même période la population diminue, il faut s'en tenir à l'évolution de la densité des patentés, donnée plus significative ; celle-ci passe de 52,68 (pour 1000 habitants) en 1851 à 47,57 au début du XXe siècle, ce qui ramène la diminution de l'artisanat à moins d'un dixième $(9,7 \%)$. Ce léger déclin du groupe des patentés est à nuancer sur le plan géographique (10). Il est plus accentué dans la partie centrale de la Beauce que dans ses marges. Ainsi dans les cantons d'Auneau, Janville et Voves - cantons situés au cœur de la Beauce la densité diminue de $15 \%$. Mais dans le même temps, dans les bourgs chef-lieux de canton, elle se maintient, comme le montre le tableau 1 .

Tab. 1 - Evolution du nombre des patentés dans les cantons d'Auneau, Janville et Voves.

\begin{tabular}{|ccc|}
\hline Années & 1851 & 1911 \\
\hline $\begin{array}{c}\text { Ensemble des cantons } \\
\text { Population }\end{array}$ & 36507 & 34119 \\
\hline Nombre de patentés & 2021 & 1605 \\
\hline Densité (pour 1 000 hab.) & 55,36 & 47,04 \\
\hline $\begin{array}{c}\text { Chef -lieux de canton } \\
\text { Population }\end{array}$ & 4269 & 5381 \\
\hline Nombre de patentés & 377 & 464 \\
\hline Densité (pour 1 000 hab.) & 88,31 & 86,22 \\
\hline
\end{tabular}


De fait, quand la population des gros bourgs augmente de $26 \%$, le nombre des patentés suit presque la même évolution : augmentation de $23 \%$. On ne peut toutefois en rester à ces constatations car la législation des patentes subit plusieurs modifications importantes au cours du XIXe siècle, modifications qui amènent à douter de la statistique des patentés, étant donnée l'augmentation progressive des exemptions (11). La confrontation avec les autres sources est donc nécessaire. Parmi les conscrits domiciliés en Beauce, la place des artisans et commerçants passe de $27,22 \%$ en 1812-1814, à $24,58 \%$ au milieu du XIXe siècle, puis à $25,42 \%$ pour les années 1905-1907. Le déclin de l'artisanat serait donc limité, et il serait surtout le fait de la première moitié $\mathrm{du}$ XIXe siècle. Il est vrai que nous considérons ici une population jeune (vingt ans) où la part du salariat est beaucoup plus forte que dans l'ensemble de la population. La profession des parents serait donc plus significative. Les listes de recrues nous l'indiquent pour des périodes limitées : premier Empire, début du XXe siècle. L'évolution vers la baisse apparaît alors plus accentuée au cours du XIXe siècle : pour le seul arrondissement de Chartres, la place des artisans et commerçants passe de $27,72 \%$ à $20 \%$ parmi les parents des conscrits (12).

Nous ferons une dernière vérification avec les listes nominatives des recensements de population. Nous les avons dépouillées pour 4 communes voisines, situées au cœur de la Beauce : Denonville, Louville-la-Chénard, Ouarville et Santeuil. Le nombre des 《 patrons » de l'artisanat et du commerce passe de 140 à 110 entre 1851 et 1901 . Par rapport au nombre d'habitants, la densité pour 1000 habitants passe de 60,37 à 56,62. Mais si l'on tient compte des aides et salariés travaillant dans ce secteur, le nombre d'actifs passe de 187 à 158 ce qui représente alors des densités respectives de 80,63 et 81,20 . En fait, on peut dire que le mouvement de baisse du nombre des artisans et commerçants accompagne celui de la population sans l'accentuer de manière significative : la part relative des artisans et commerçants a peu diminué au cours du XIXe siècle (13). Les modifications affectent beaucoup plus la diversité interne de cette couche sociale que son importance globale.

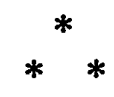

Analyser les structures de l'artisanat et du commerce suppose d'abord l'établissement d'un classement des secteurs d'activité et des métiers, réalisé à partir des Registres de renseignements statistiques et administratifs des contrôles des contributions directes. Il est souvent difficile, à partir de cette documentation, de séparer, parmi les patentés, les deux activités artisanale et commerciale. Dans la réalité, la plupart des artisans vendent leur production ou le fruit de leur travail. De même beaucoup de commerçants - surtout dans le petit commerce - ont une part d'activité directement productive. Nous adopterons donc un classement par secteurs d'activité assez détaillé pour permettre des regroupements différents (tableau 2). 
Tab. 2 - Répartition par secteurs d'activité et évolution des parentés de 1846 à 1879 dans les quatre cantons d'Auneau, Chartres-Sud, Janville et Voves.

\begin{tabular}{|c|c|c|c|c|c|}
\hline \multirow[t]{2}{*}{ Secteurs d'activité } & \multicolumn{2}{|c|}{1846} & \multicolumn{2}{|c|}{1879} & \multirow{2}{*}{$\begin{array}{c}\text { Evolution } \\
1846-1879 \\
\text { (en \%) }\end{array}$} \\
\hline & Nombre & $\%$ & Nombre & $\%$ & \\
\hline \multicolumn{6}{|l|}{ COMMERCE } \\
\hline - Céréales & 14 & 3 & 34 & 6,4 & $+142,3$ \\
\hline - Bestiaux & 89 & 19,2 & 87 & 16,4 & $-\quad 2,2$ \\
\hline - Produits agricoles & 51 & 11 & 53 & 10 & $+3,9$ \\
\hline - Produits alimentaires & 55 & 11,8 & 83 & 15,7 & $+50,9$ \\
\hline - Textiles & 108 & 23,3 & 73 & 13,8 & $-32,4$ \\
\hline - Divers & 146 & 31,5 & 199 & 37,6 & $+36,3$ \\
\hline Ensemble du commerce & 463 & 100 & 529 & 100 & $+14,25$ \\
\hline \multicolumn{6}{|l|}{ ARTISANAT } \\
\hline - Bois & 321 & 16,6 & 125 & 8 & -61 \\
\hline- Textile & 113 & 5,8 & 58 & 3,7 & $-48,7$ \\
\hline- Fer & 179 & 9,2 & 115 & 7,4 & $-35,7$ \\
\hline - Bâtiment & 284 & 14,7 & 268 & 17,2 & $-\quad 5,6$ \\
\hline - Alimentation & 405 & 20,9 & 422 & 27 & $+4,2$ \\
\hline - Cuir & 193 & 10 & 77 & 4,9 & $-60,1$ \\
\hline - Transport & 18 & 0,9 & 50 & 3,2 & $+177,7$ \\
\hline - Sociabilité (1) & 421 & 21,8 & 444 & 28,5 & $+5,5$ \\
\hline Ensemble de l'artisanat & 1934 & 100 & 1559 & 100 & $-19,40$ \\
\hline DIVERS & 56 & & 135 & & \\
\hline TOTAL & 2453 & & 2223 & & $-\quad 9,38$ \\
\hline
\end{tabular}

(1) Il s'agit des cafetiers, cabaretiers, aubergistes.

Dans les 4 cantons de la pleine Beauce que nous avons considérés, la part du commerce est minoritaire en $1846(18,87 \%)$ comme en $1879(23,79 \%)$. Même si l'on inclut dans le commerce les artisans de "sociabilité » (cabaretiers, aubergistes) le constat est le même, l'activité commerciale est moins importante que l'activité proprement artisanale : $36,03 \%$ en $1846,43,76 \%$ en 1879 . On remarque en outre que parmi les patentés, cette activité commerciale progresse de $14 \%$ entre 1846 et 1879 , alors que le nombre des artisans baisse de $19 \%$. En éliminant le secteur sociabilité de ceux-ci, la baisse serait encore plus importante : $26,30 \%$. Sans doute faut-il nuancer cette conclusion, et ne pas exagérer ces mouvements, car les modifications de la législation des patentes contribuent à exempter beaucoup plus les métiers de l'artisanat que ceux du commerce (11). Mais les autres sources - recensements et listes des recrues - montrent également une évolution analogue.

$\mathrm{Au}$ niveau du commerce, mis à part les marchands divers (il s'agit surtout du commerce non spécialisé, essentiellement de marchands forains dont le nombre passe 
de 79 en 1846 à 101 en 1879), le commerce des produits agricoles au sens large (céréales, bestiaux et autres) occupe la première place, représentant le tiers des marchands. Dans l'ensemble, il progresse en nombre, surtout pour l'écoulement des céréales dont la production augmente au cours de cette période. D'autre part le nombre des blatiers et marchands de blé s'accroît pendant cette période en liaison avec l'établissement de nouveaux circuits commerciaux : les cultivateurs vendent de plus en plus sur échantillons et les quantités livrées sur les marchés commencent à décliner fortement. La progression du commerce alimentaire est à mettre en rapport avec l'élévation incontestable du niveau de vie, dont témoigne également la catégorie " divers » qui traduit un élargissement et une diversification des produits offerts aux habitants. Par contre on soulignera le déclin important du commerce textile, déclin qui se retrouve au niveau artisanal de ce secteur économique. On touche là à une difficulté quant à la définition de l'artisanat, car il s'agit en fait d'une "industrie » à domicile employant surtout des femmes payées aux pièces par des 《 marchands-fabricants 》 orléanais ou chartrains qui fournissent la matière première (laine) pour la fabrication d'articles de bonneterie. D'après une description géographique de l'Eure-et-Loir publiée en 1836, dans le canton de Voves, « on fabrique des bas, bonnets et gilets de laine au tricot : il y a au moins 2000 femmes employées à ce travail excepté pendant le temps de la moisson (soit le sixième de la population) »(14). Dans la commune d'Oisonville (canton d'Auneau) 3 fabriques occupent 40 hommes, 300 femmes et enfants. Dans le canton de Janville, la même source cite le chiffre de 3000 femmes et enfants qui tricotent la laine. Cette activité s'est fortement réduite au milieu du XIXe siècle, comme l'indique par exemple la monographie du canton d'Auneau effectuée par le contrôleur des contributions directes vers 1850 :

"Les habitants de 4 ou 5 communes, étaient autrefois activement occupés à la fabrication des bas, chaussons, bonnets et gants de laine, mais cette branche d'industrie étant tombée depuis quelques années, ne fait plus que végéter ; le commerce des laines et moutons qui sont très abondants dans cette partie de la Beauce a seul conservé son activité primitive. » (15)

Cette bonneterie à l'aiguille est donc en voie de disparition au milieu du XIXe siècle. Son déclin explique sans doute les difficultés que connaissent les classes populaires dans les années 1830-1840, difficultés qui se traduisent par la montée de la mendicité, avant d'être résolues en partie par l'exode rural qui touchera en premier lieu le prolétariat rural. La conséquence en est également la diminution de l'artisanat et du commerce textile liés à cette « industrie » rurale.

Dans l'artisanat, globalement en recul selon la statistique des patentés, certains secteurs se maintiennent ou progressent, notamment ceux qui sont en rapport avec une activité également commerciale : alimentation, transport et sociabilité. Par contre tous les autres métiers perdent de leur importance, sauf le secteur du bâtiment. Mais étant donné les exemptions qui suivent les modifications de la législation des patentes on peut s'interroger sur la valeur de ces tendances. L'étude attentive des professions indiquées sur les listes nominatives pour les 4 communes de la pleine Beauce que nous avons déjà évoquées montre qu'il y a plutôt stabilité relative des différents corps de métier, hormis le déclin vérifié du textile (tableau 3). 
Tab. 4 - Classement administratif des parentés du tableau A dans les quatre cantons d'Auneau, Chartres-sud, Janville et Voves.

\begin{tabular}{|c|c|c|c|c|c|c|c|c|}
\hline \multirow{3}{*}{ CLASSES } & \multicolumn{4}{|c|}{ Ensemble des quatre cantons } & \multicolumn{4}{|c|}{ Communes de plus de 1000 hab. } \\
\hline & \multicolumn{2}{|c|}{1846} & \multicolumn{2}{|c|}{1879} & \multicolumn{2}{|c|}{1846} & \multicolumn{2}{|c|}{1879} \\
\hline & Nombre & $\%$ & Nombre & $\%$ & Nombre & $\%$ & Nombre & $\%$ \\
\hline A $1,2,3$ & 145 & 6,7 & 122 & 6,8 & 69 & 10,4 & 64 & 10,2 \\
\hline A4 & 319 & 14,7 & 345 & 19,4 & 120 & 18,1 & 161 & 25,7 \\
\hline $\mathrm{A} 5,6,7,8$ & 1709 & 78,6 & 1310 & 73,7 & 474 & 71,4 & 401 & 64,1 \\
\hline TOTAL & 2173 & & 1777 & & 663 & & 626 & \\
\hline
\end{tabular}

$\mathrm{Au}$ total, il y a en Beauce, dans la deuxième moitié du XIXe siècle une diminution de l'artisanat et un progrès certain de l'activité commerciale. Les métiers les plus nombreux ont un rapport étroit avec l'activité commerciale : aubergistes, cabaretiers, commerce alimentaire, épiciers. L'artisan au service du cultivateur - bourrelier, charron, maréchal-ferrant - garde sans doute sa place sans grande modification tout au long du siècle. C'est pourquoi tous les témoignages concordent pour souligner encore au début du XXe siècle la forte densité artisanale dans les plus modestes villages. Ainsi dans le village de Moutiers-en-Beauce qui atteignait tout juste 400 habitants en 1911:

" y'avait trois meuniers, deux boulangers, quatre bistrots, deux charrons, deux maréchaux, deux menuisiers, deux cordonniers, un tailleur d'habits, un marchand d'moutons. Tout ça travaillait, aujourd'hui ça travaillerait pus $"$. (16)

L'absence de transformation majeure dans les techniques agricoles suffit à expliquer cette stabilité : toute une série de métiers est liée à l'utilisation du cheval comme principale force de travail (bourreliers, maréchaux, charrons). Chaque grande ferme dispose d'une dizaine de chevaux, et la quasi-totalité des instruments de travail peut être entretenue et réparée au village. C'est ce qui explique l'importance des notes payées chaque année par les cultivateurs aux artisans. Une enquête faite en 1909 dans six grandes fermes de la Beauce (17) montre qu'en année moyenne la grande ferme consacre près de $6 \%$ de ses dépenses - soit entre 2000 et 3000 francs - en notes du charron, du maréchal, du bourrelier et autres artisans. Ce n'est qu'avec la généralisation du tracteur que ces métiers disparaîtront. Dans les autres métiers, le bâtiment reste au même niveau tout en se concentrant : moins de patrons, davantage de salariés. La concentration touche également les meuniers, surtout ceux qui exploitent des moulins à vent. D'une manière générale, commerce et travail du textile quittent la campagne beauceronne après le milieu du XIXe siècle. L'impression dominante, une fois prises en compte les limites des différentes sources, est la stabilité des secteurs liés au travail de la terre, le déclin du textile et le progrès du commerce alimentaire lié à l'élévation du niveau de vie. Les artisans au service du consommateur (cuir, bois, bâtiment) se maintiennent tout en se concentrant dans certains domaines.

Mais par delà l'évolution respective des différents métiers, il importe de tenter une approche de stratification interne au milieu artisanal et commercial. La différenciation ne se fait pas seulement par la nature du travail, la part du travail productif 
et des opérations commerciales. Au sein d'un même métier il peut y avoir des niveaux très différents, des " chiffres d'affaires 》 et des profits que l'on devine très éloignés. Il suffit pour en avoir conscience de regarder la vie que mènent certains témoins ou accusés de ce secteur économique interrogés lors des procès d'Assises. Ainsi, si l'on prend le commerce de moutons, on peut avoir, d'après les dossiers d'Assises de 1857, deux situations aux antipodes. Au plus modeste, il y a le commerce de moutons de rebut associé à la boucherie et exercé par $\mathrm{A}$. Lamé et sa mère demeurant à Bailleausous-Gallardon et dont l'activité est ainsi décrite par le juge de paix d'Auneau, le 8 janvier 1857 : «Le père de Lamé faisait le commerce de moutons de rebut. Depuis son décès il y a environ 2 ans, la Veuve $L$. a continué avec l'aide... de son fils qui court les marchés et les campagnes, achète et tue les animaux qu'il ramène et que sa mère dépèce pour les vendre en détail dans les communes rurales et même quelquefois à Auneau. » (18)

C'est une activité de maigre rapport puisque la veuve Lamé reconnaît que son « mari était inscrit à la charité publique ; quant à moi je vivais de mon travail de journalière » (18). Rien de comparable avec les affaires brassées dans le même domaine au début du Second Empire par G. Rabourdin, fermier et marchand de moutons demeurant à Nogent-le-Phaye. Les cultivateurs évoquent ainsi le personnage : «Depuis environ 3 ou 4 ans j'ai été en relation d'affaires avec Monsieur Rabourdin. Je lui ai vendu plusieurs fois des moutons, il les a toujours payés comptant. Sans en être certain, je le crois dans une bonne position de fortune, car pour faire son commerce il a besoin de fonds considérables, attendu que les moutons se payent comptant aux cultivateurs et que ceux à qui il les livre dans les marchés ne payent en général qu'à terme » (19). Un autre ajoute : " depuis 10 à 12 ans je connais Rabourdin et je fais des affaires avec lui ; il dirige une grande exploitation et fait un commerce considérable sur les moutons... Rabourdin est le plus fort commerçant que je connaisse... Il a la confiance des marchands et pourrait avoir auprès d'eux, s'il le voulait, un crédit illimité » (19).

Pour situer ces différents niveaux à l'intérieur de l'artisanat et du commerce, nous pouvons utiliser deux critères, celui de la valeur locative et celui des fortunes au mariage.

Tab. 3 - Evolution des secteurs d'activité artisanale d'après les listes nominatives des communes de Denonville, Louville-la-Chénard, Ouarville et Santeuil.

\begin{tabular}{|lrrrr|}
\hline \multicolumn{2}{|c}{1851} & \multicolumn{2}{c|}{1901} \\
& Nombre & $\%$ & Nombre & $\%$ \\
\hline Bois & 27 & 20,7 & 20 & 23,5 \\
\hline Textile & 11 & 8,5 & 3 & 3,5 \\
\hline Fer & 11 & 8,5 & 10 & 11,6 \\
\hline Bâtiment & 21 & 16,1 & 15 & 17,4 \\
\hline Alimentation & 25 & 19,2 & 17 & 11,6 \\
\hline Cuir & 16 & 12,3 & 10 & 11,6 \\
\hline Transport & 1 & & 1 & \\
\hline Sociabilité & 18 & 13,8 & 10 & \\
\hline \multicolumn{1}{c}{ ENSEMBLE } & 130 & & 86 & \\
\hline
\end{tabular}


L'administration financière a tenté de saisir ces différents niveaux au sein d'une même activité par l'intermédiaire des classes de patentés à l'intérieur de plusieurs tableaux (A, B, C, D). C'est ainsi que les 3 premières classes du tableau A regroupent les gros commerçants ; les 5 dernières classes (A4 à A8) peuvent être assimilées au commerce de détail et à l'artisanat. Selon son chiffre d'affaires, l'importance de son activité, sa valeur locative un artisan pourra être placé dans une classe ou une autre : un maçon entrepreneur sera en A4, un maître-maçon en A6 et un maçon en bauge en A7. En gros, on peut dire que quand on passe de A1 en A7 ou A8, le niveau d'activité, de revenus diminue nettement. On peut donc utiliser ce classement pour avoir une idée grossière du gros et moyen commerce (A1 à A4) et du petit commerce et de l'artisanat. Pour les 4 cantons spécifiquement beaucerons déjà considérés (tableau 4) le gros commerce - dont la valeur locative moyenne est de 244,5 francs en 1846 par patenté - est très minoritaire. Il regroupe les marchands de vin, beurre, bois, laine, tissus. Son importance s'accroît nullement entre 1846 et 1879 : il reste autour de 6 à $7 \%$ des patentés de la classe A. Par contre sa place est plus grande dans les communes les plus peuplées $(10 \%)$. Le moyen commerce (A4) qui en est proche (valeur locative moyenne de 221 francs en 1846) et qui regroupe les aubergistes, les bouchers, charcutiers, cafetiers et marchands de bestiaux ainsi que les artisans les plus importants, joue un rôle plus grand et en augmentation pendant la deuxième moitié du XIXe siècle. L'artisanat et le petit commerce sont nettement prépondérants, avec environ les $3 / 4$ des patentés. Il est à noter que l'artisanat et le commerce le plus modeste, dispensé du paiement de droits proportionnels - et pour lequel nous ignorons donc la valeur locative alors que pour les classes A5 et A6 elle est en. moyenne de 117 francs en 1846, ce qui montre bien la différence de niveau avec les classes supérieures dépassant 220 francs - tend à diminuer nettement (de 20 à $12 \%$ du tableau $\mathrm{A}$ entre 1846 et 1879) du fait des exemptions nouvelles. Comme il est attendu, le commerce de gros se concentre dans les communes les plus importantes, les gros bourgs, mais ceux-ci n'en ont pas l'exclusivité ; par exemple, en 1879, sur 43 marchands de vins en gros, il y en a 20 qui demeurent dans les communes de moins de 1000 habitants.

Ce classement administratif se limite au tableau A et ignore ainsi certaines professions, inscrites au tableau $\mathrm{C}$, comme les meuniers, les marchands-forains, etc. A défaut de la matrice des patentes, les Registres de renseignements statistiques et administratifs des contrôleurs des contributions directes qui indiquent par commune et pour chaque profession le montant total des valeurs locatives, nous donnent la possibilité d'avoir une idée des valeurs locatives respectives des différents métiers. En ne retenant que les professions ayant une représentation numérique suffisante (tableau 5) on doit constater que, dans l'ensemble, l'activité artisanale a une valeur locative moyenne supérieure à celle du commerce. Toutefois ces moyennes effacent les différences de niveau à l'intérieur de chaque métier ; d'autre part certains métiers comptés dans l'artisanat (aubergistes, meuniers) ont une très forte valeur locative qui peut fausser le calcul d'une moyenne d'ensemble propre à chacun des deux secteurs, artisanat et commerce ; enfin, on admettra que l'administration avait sans doute une plus grande facilité pour estimer la valeur locative d'un atelier (maréchalerie, moulin par exemple) alors que l'estimation pouvait être sous-estimée pour certains commerces ne nécessitant pas de boutique (commerce de produits agricoles, bestiaux etc.). Dans ces conditions il faut être prudent dans les conclusions que l'on peut tirer à partir de ce critère. Ce sont les meuniers qui dominent la société artisanale. Aussi les retrouve-t-on nombreux sur les listes électorales censitaires. Les plus importants sont ceux qui possèdent ou 
Tab. 5 - Evolution de la valeur locative moyenne (en francs) dans quatre cantons beaucerons de 1846 à 1879 .

(Auneau, Chartres-sud, Janville et Voves).

\begin{tabular}{|lccc|}
\hline \multicolumn{1}{c}{ METIERS } & 1846 & 1879 & Évolution en $\%$ \\
\hline Marchands de bestiaux & 127 & 204 & $+60,6$ \\
\hline Marchands de vins en gros & 291 & 259 & -11 \\
\hline Marchands de tissus & 290 & 285 & $-1,7$ \\
\hline Marchands-forains & 103 & 149 & $+44,6$ \\
\hline Charpentiers & 111 & 151 & +36 \\
\hline Charrons & 113 & 163,5 & $+44,2$ \\
\hline Menuisiers & 122 & 132 & $+8,2$ \\
\hline Maréchaux & 132 & 138 & $+4,9$ \\
\hline Marchands-quincaillers & 379 & 493 & +30 \\
\hline Serruriers & 160 & 174,5 & $+2,7$ \\
\hline Maçons & 83 & 100 & $+20,5$ \\
\hline Meuniers & 607 & 621 & $-7,3$ \\
\hline- dont meuniers à vent & 416 & 383 & $+21,2$ \\
\hline- dont meuniers à eau & 1284 & 1556 & $+47,3$ \\
\hline Boulangers & 169 & 249 & $+44,6$ \\
\hline Bouchers & 166 & 240 & +27 \\
\hline Bourreliers & 118 & 150 & $+6,1$ \\
\hline Cordonniers & 131 & 139 & $+26,2$ \\
\hline Aubergistes & 305 & 324 & $+17,5$ \\
\hline Cabaretiers & 117 & 148 & $+23,6$ \\
\hline Ensemble des ARTISANS & & & \\
\hline Ensemble des COMMERCANTS & 206 & 242 & \\
\hline
\end{tabular}

travaillent dans un moulin situé sur l'Eure (canton de Chartres-sud). Mais les exploitants de moulins à vent dépassent également les autres métiers, même si le déclin de ce genre de meunerie se traduit par une baisse de la valeur locative des moulins. Viennent ensuite - en dehors des marchands-quincaillers, peu nombreux - les aubergistes, les commerçants du textile, les marchands de vins en gros. A un degré inférieur, on trouve les artisans de l'alimentation (en plein essor comme le montre la forte hausse de leur valeur locative), les marchands de bestiaux. En général les métiers du bois, du fer, du cuir et du bâtiment sont au bas de l'échelle des valeurs locatives. Au sommet le meunier ; à la base, le maçon ? On pourrait simplifier ainsi les différences au sein de l'artisanat, en passant par le commerce chargé d'écouler les productions agricoles, le commerce alimentaire, puis la masse de ceux qui travaillent pour les cultivateurs et les consommateurs. L'incertitude de la notion de valeur locative laisse toutefois un doute sur ces conclusions : on ne peut comparer l'absence d'atelier du maçon, la faiblesse de son outillage à la valeur vénale et locative d'un moulin à vent ou d'une auberge. Ne risque-t-on pas d'avoir une hiérarchie à l'image des dimensions matérielles impliquées par chaque métier ? Il est vrai toutefois que c'est à l'image du capital engagé et en ce sens la hiérarchie proposée présente son intérêt. De ce point de vue, l'analyse des fortunes dont disposent les artisans au moment du mariage apporte d'utiles comparaisons. 
Comme l'âge moyen au mariage se situe entre vingt et trente ans nous pouvons considérer les apports au mariage comme un indice valable du niveau de capital nécessaire à l'exercice du métier. Nous avons relevé tous les mariages qui ont eu lieu pendant les années 1811 à 1820 dans le canton de Chartres-Sud (20). Nous nous sommes ensuite reportés aux Tables des contrats de mariage du bureau de Chartres de l'Enregistrement (21) pour relever ceux de ces mariages qui étaient accompagnés de contrats : pour environ 300 mariages d'artisans il y a 137 contrats signés, c'est-àdire que près d'un artisan sur deux, au début du XIXe siècle, se marie en allant chez le notaire. On peut donc considérer ces apports au mariage comme représentatifs de la fortune des artisans eu début de leur établissement, étant donné la fréquence de ces contrats. Pour constituer un échantillon valable, les apports des conjoints artisans - et pour comparaison ultérieure, des cultivateurs et des salariés agricoles - ont été relevés pour la décennie 1811-1820 dans trois autres cantons beaucerons (Auneau, Janville et Voves). Pour 528 ménages d'artisans la valeur moyenne de la fortune au mariage est de 1824 francs (tableau 6). Pour les 87 ménages de commerçants recensés - en incluant les commerçants-artisans de l'alimentaire : bouchers, boulangers, aubergistes, pour compenser la faiblesse de l'échantillon - la valeur moyenne est de 6565 francs. Au début du XIXe siècle, les commerçants sont donc nettement plus fortunés que les artisans, contrairement à ce qu'indiquait la valeur locative. Il est probable que celle-ci mesure plus l'importance de la boutique en tant que bâtiment, alors que la fortune au mariage représente en plus le capital nécessaire à la formation du stock de marchandises. Parmi les métiers, on retrouve toujours, au sommet de l'artisanat, les meuniers, mais ils sont suivis de près par les maréchaux. Viennent ensuite les charrons, cordonniers, artisans du textile, et, au bas de l'échelle des fortunes, comme de celle des valeurs locatives, les maçons. Mais l'étude du niveau des fortunes montre que même pour les métiers du haut de l'échelle - meuniers, maréchaux - l'écart est important entre les moins fortunés et ceux qui sont au sommet de la hiérarchie. Les clivages traversent les genres d'activité identiques. C'est pourquoi le classement de l'administration financière en tableaux et classes apparaît judicieux. Il existe bien un gros et moyen commerce (classes Al à A4) dont la valeur locative peut être trompeuse

Tab. 6 - Fortunes moyennes au mariage en 1811-1820

(d'après les Tables des contrats de mariage

des bureaux d'Auneau, Janville et Voves de l'Enregistrement).

\begin{tabular}{|lrc|}
\hline \multicolumn{1}{|c}{ Catégories sociales } & Nombre de cas & $\begin{array}{c}\text { Fortunes moyenne } \\
\text { (en francs) }\end{array}$ \\
\hline Cultivateurs & 327 & 8524,25 \\
\hline Domestiques agricoles & 459 & 890,65 \\
\hline Journaliers & 259 & 830,83 \\
\hline Vignerons & 63 & 1066,74 \\
\hline Artisans & 528 & 1824,14 \\
\hline Commerçants & 87 & 6565,73 \\
\hline Détail des artisans : & & \\
\hline - maçons & 125 & 986,39 \\
\hline- charrons & 45 & 1897,88 \\
\hline- maréchaux & 47 & 2972,17 \\
\hline- textile & 119 & 1267,42 \\
\hline- cordonniers & 53 & 1574,71 \\
\hline- meuniers & 45 & 3000,51 \\
\hline
\end{tabular}


(il suffit de penser au commerce de céréales, de bestiaux) mais qui domine nettement, par les capitaux engagés, suggérés par la fortune au mariage, la masse du petit commerce et de l'artisanat, excepté les meuniers et certains maréchaux qui sont plus proches du gros commerce que des artisans les plus modestes, à savoir les maçons. On pourrait ainsi schématiser le milieu de l'atelier et de la boutique en Beauce au milieu du XIXe siècle : un cinquième appartiendrait plutôt à la bourgeoisie - et la place de celle-ci tend à augmenter au cours du siècle -, les quatre-cinquièmes relèvent plutôt des classes populaires, mais pas réellement du prolétariat.

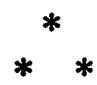

En effet l'analyse des artisans dans leurs rapports avec les autres classes de la société rurale montre qu'on ne peut vraiment les rattacher au salariat, même s'ils s'en rapprochent par beaucoup d'aspects.

Dans la société beauceronne, l'artisan apparaît d'abord comme étroitement lié à la terre. Pour une large part, nous l'avons vu, les cultivateurs constituent un de ses principaux débouchés. Les commerçants qui assurent la vente des productions agricoles (marchands de blé, de bestiaux...) représentent le tiers de l'ensemble du commerce. Ils font généralement partie du gros et moyen commerce. En comptant les artisans travaillant directement pour les fermes (charrons, bourreliers, artisans du fer) on a près d'un cinquième du milieu considéré qui est au service direct des cultivateurs, de la production agricole.

Beaucoup d'artisans sont en même temps propriétaires, cultivateurs et une part de leurs revenus a pour origine le travail de la terre. Si l'on prend par exemple le canton de Voves, au moment de la confection du Cadastre (1836) le milieu de l'atelier et de la boutique possède $17,7 \%$ de la terre que contrôlent les beaucerons. Or d'après le recensement de 1851 , artisans et commerçants représentent $17,2 \%$ de la population active masculine dans ce canton. On voit que les artisans - et surtout les commerçants, plus riches de ce point de vue - possèdent une partie de la terre équivalente à leur place dans la population. La terre forme une part essentielle de leur fortune. Ainsi dans l'échantillon d'artisans qui se sont mariés entre 1811 et 1820 dans le canton de Chartres-Sud, nous trouvons, pour 153 d'entre eux dont nous avons pu relever la déclaration de succession après décès, que la fortune immobilière représente 74,80\% de la fortune totale, dont $19 \%$ en bâtiment et $55,80 \%$ en terre proprement dite. En règle générale l'artisan a ainsi une petite culture. Presque tous les artisans ont des bâtiments à usage agricole : grange, étable. La femme s'occupe d'une vache, du petit bétail. Au niveau le plus modeste, l'artisan peut s'embaucher comme moissonneur ou journalier dans les grandes fermes pendant les grands travaux de l'été.

Travaillant essentiellement pour l'agriculteur - plus en tant que consommateur que producteur - paysan lui-même, certes modestement, l'artisan beauceron est-il réellement indépendant? Entre le salariat et les cultivateurs, quelle est sa place ?

$\mathrm{Au}$ niveau du travail, certains artisans peuvent être assimilés à des journaliers. Charrons, bourreliers, charpentiers, menuisiers travaillent sur place, dans les fermes. 
Ils y sont parfois nourris, comme les domestiques agricoles. Pour certains le paiement se fait à la journée. Autant dire que leur statut est presque celui d'un salarié ! Travaillant seuls, à façon, ils sont de fait ou de droit exemptés de patente. Tel est le cas exemplaire de Jean Langlois menuisier à Santilly accusé en 1857 de vol chez le cultivateur qui l'employait (22). La fermière décrit ainsi son activité : «Langlois travaille pour nous depuis qu'il est à Santilly, c'est-à-dire depuis environ huit ans, mais jusqu'au 15 avril de l'année dernière, il n'avait fait pour nous que fort peu de chose et je l'avais toujours payé à la journée... » L'acte d'accusation précise qu'il «était fréquemment employé à la ferme dont il était l'hôte et le familier ; il y avait un lit... » et ajoute qu'il n'avait pour toute ressource qu'un salaire de 2 francs par jour. Langlois ne paie plus de patente : "cette exonération a eu lieu sur la demande même du percepteur qui n'a jamais pu recouvrer sur lui les contributions qu'il devait... »

Ce cas montre l'existence d'une plèbe artisanale qui se confond avec la classe des journaliers. Alors la dépendance est complète à l'égard du client devenu employeur. Ces cas peuvent se rencontrer parmi les métiers ne nécessitant qu'un faible outillage, qu'un capital très limité (bâtiment, travail du bois, du cuir). Ils sont beaucoup plus rares parmi ceux qui ont besoin d'un capital plus important lié à la présence obligatoire d'un atelier ou d'un boutique (forge, moulin, auberge). Alors l'indépendance peut être acquise. Autrement dit, plus la part du travail augmente, plus le capital apparait faible, plus on se rapproche des classes populaires. Inversement, quand le capital utilisé est élevé, l'artisan et surtout le commerçant font partie de la bourgeoisie.

C'est ce que confirme l'analyse des fortunes. Les listes d'électeurs censitaires de 1846 pour les 3 cantons beaucerons d'Auneau, Janville et Voves indiquent la présence de 80 patentés soit $21,8 \%$ de l'électorat. Les fermiers représentent alors $30 \%$ des électeurs. Dans ces 3 cantons il y a, en 1846, 1924 patentés. Ce sont donc seulement $4 \%$ des artisans et commerçants qui peuvent devenir électeurs, alors que l'on peut estimer à $60 \%$ le nombre des exploitants des grandes fermes qui paient assez d'impôt (200 francs de cens) pour être électeurs. Dans son ensemble l'artisanat paraît donc loin derrière la grande culture dans l'échelle des fortunes rurales. Les patentés censitaires sont surtout des représentants du gros et moyen commerce, à valeur locative élevée (meuniers, négociants en vins, aubergistes). Ce clivage entre commerce et artisanat se retrouve au niveau des fortunes au mariage (tableau 6). La fortune moyenne d'un cultivateur, au début du XIXe siècle est supérieure à 8000 francs. Celle des salariés agricoles - domestiques et journaliers - est située entre 800 et 900 francs ; on sait qu'au mariage l'artisan dispose d'environ 1800 francs, le commerçant de 6500 francs. Par delà les moyennes, la répartition par niveaux prouve que l'artisan est très éloigné du niveau d'aisance du cultivateur, alors qu'une partie du commerce a une fortune comparable au milieu de la grande culture. Néanmoins les artisans ne peuvent être assimilés aux salariés : leurs apports au mariage sont fréquemment plus élevés, en moyenne de plus du double. Il y a donc un niveau de fortune propre au milieu artisanal qui le distingue dans son ensemble des classes populaires, ce qui va dans le sens d'une certaine autonomie de ce milieu social.

La même impression se retrouve quand on essaie de saisir l'homogénéité du milieu artisanal à travers le critère du choix des épouses lors du mariage. Nous connaissons - pour l'échantillon constitué dans le canton de Chartres-Sud entre 1811 et 1820 les professions des parents des épouses pour 301 mariages d'artisans et commerçants. 
Dans $35 \%$ des cas, le choix de l'épouse se fait dans le même milieu social ; dans $23 \%$ des cas l'épouse est fille d'ouvriers agricoles ; les cultivateurs et les vignerons fournissent respectivement 23 et $14 \%$ des épouses d'artisans. L'apport au mariage des filles de cultivateurs est très moyen, autour de 1400 francs pour les mariages avec les artisans, plus élevé pour les mariages avec les commerçants : 3000 francs environ. En regard des fortunes moyennes au mariage, il apparaît donc qu'il n'y a pas d'ascension sociale par le mariage : on se marie en rapport avec sa situation sociale et sa position de fortune. Ce qui place les artisans au niveau d'une importante couche de petits cultivateurs, de vignerons (coteaux de la vallée de l'Eure pour ce canton de ChartresSud) c'est-à-dire d'une petite bourgeoisie rurale qui peut se rapprocher dans ses éléments inférieurs du salariat : près d'une épouse sur quatre vient de ce milieu. On a donc une classe intermédiaire, indépendante, entre le prolétariat agricole et le groupe des grands cultivateurs capitalistes, étant donné qu'elle est plus proche - sauf quelques gros commerçants, meuniers et aubergistes - du premier que du second. Les artisans se situent au même niveau social que les petits cultivateurs avec lesquels ils ont de fréquentes relations. Mais une partie inférieure de l'artisanat, par ses formes de travail, la faiblesse de son capital, se confond pratiquement avec les classes populaires. Aussi ne faut-il pas s'étonner de constater que si l'artisanat se recrute pour une part parmi les salariés, il ne permette guère d'accéder à un niveau social supérieur.

On peut en effet à partir de l'échantillon constitué, faire une analyse de mobilité sociale. Pour les 301 mariages dont nous connaissons la profession du père de l'époux, $48 \%$ des fils exercent le même métier que le père, et près de $60 \%$ sont fils d'artisans ou commerçants. Le recrutement interne est donc prépondérant. Pour une faible part les artisans sont originaires de la petite culture, comme l'indique la faiblesse de l'apport au mariage dont ils disposent. Enfin $20 \%$ des artisans sont des enfants d'ouvriers agricoles dont l'apport au mariage est faible et ne nécessite pas le plus souvent de contrat de mariage.

Originaire en grande partie de la même petite bourgeoisie l'artisan rural a-t-il des chances de s'enrichir au cours de sa vie, dans l'exercice de son métier ? Nous avons recherché dans les archives de l'Enregistrement la situation de fortune, au moment du décès, des ménages constitués en 1811-1820, représentatifs d'une génération née à la fin du XVIIIe siècle (23) et dont la mort se situe à l'époque du Second Empire. $\mathrm{La}$ situation de fortune au décès a été retrouvée pour 213 cas, pour lesquels nous pouvons comparer professions et niveaux de fortune au mariage et à la fin de la vie. Un bon tiers garde la même profession tout au long de sa vie. La moitié environ sont qualifiés - dans les registres de mutations par décès - de rentiers, propriétaires, sans profession, ou d'anciens artisans. Il s'agit en fait d'artisans et de commerçants retirés, qui se sont assurés par leur activité les moyens d'une honnête retraite. Le déclassement est limité : 28 artisans, soit $13 \%$ du total sont qualifiés à leur mort de journaliers. Très rares sont les artisans devenus agriculteurs ou même commerçants. Le déclassement peut toucher également le commerce : sur 30 commerçants dont nous connaissons la situation au décès, 6 sont devenus salariés. Le critère professionnel étant peu sûr, il est plus intéressant de comparer fortune au mariage et fortune au décès et de calculer ainsi un taux annuel moyen d'enrichissement ou... d'appauvrissement. On constate que sur 213 artisans et commerçants, 33 meurent indigents, sans laisser aucun bien, soit près de $15 \%$. Parmi les 180 artisans et commerçants dont nous connaissons la fortune au moment de la mort, 107 avaient rédigé un contrat de mariage. Pour une durée moyenne de 40 ans, le taux d'enrichissement annuel est de 5,6\% (24). 
C'est-à-dire qu'en se mariant au début du XIXe siècle, un artisan pouvait espérer, lors de son décès à l'époque du Second Empire, avoir plus que triplé la fortune dont il disposait au mariage. Mais ce taux moyen cache des situations extrêmes, car plus $\mathrm{du}$ tiers ont vu au contraire leur fortune diminuer, alors qu'un quart ont pu dépasser un gain annuel de $10 \%$... Il y a donc une très grande inégalité de réussite sociale au sein du milieu artisanal. De même l'analyse de la profession des enfants héritiers - signalée trop rarement dans les registres de mutations par décès - montre que des cas extrêmes peuvent se rencontrer : le fils d'artisan est souvent un salarié, très rarement il est qualifié de commerçant. Plus de la moitié des fils d'artisans dont nous connaissons la profession restent artisans. Par delà une stabilité majoritaire, le mouvement de descente sociale est beaucoup plus représenté que celui de l'ascension sociale. On a ainsi une nouvelle confirmation de la place de l'artisanat au sein de la société rurale beauceronne.

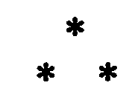

Faisant travailler le quart de la population active au milieu du XIXe siècle, légèrement moins au début du XXe siècle, mais sans diminution significative, l'artisanat et le commerce sont au service de l'agriculture en Beauce et ils sont relativement intégrés à la société rurale non seulement par les débouchés mais également par le travail de la terre. C'est ce qui explique, avec l'absence de transformation majeure dans les techniques agricoles, l'élargissement des débouchés du à l'élévation du niveau de vie, entre autres, le maintien tout au long du XIXe siècle d'un nombre aussi élevé d'artisans et de boutiquiers pour une région rurale.

Toutefois cette intégration en milieu rural n'est pas synonyme de dépendance, et la majorité des artisans et commerçants constitue une classe intermédiaire entre le prolétariat agricole et les grands fermiers qui représentent en Beauce le capitalisme agricole. La possession d'un capital, le travail de la terre, le niveau des fortunes comme les échanges matrimoniaux situent cette classe au même niveau que celle des petits cultivateurs et l'on peut alors parler de petite bourgeoisie rurale.

Mais sa position intermédiaire en fait aussi une classe hétérogène. D'une manière générale, l'activité commerciale minoritaire, mais en progrès au cours du XIXe siècle, se rapproche d'une bourgeoisie " moyenne ", comme le montrent l'importance des fortunes au mariage, l'accès de certains marchands à l'électorat censitaire, et comme le consacre le classement de l'administration financière (les premières classes du tableau A). Par contre, l'immense majorité de l'artisanat et du petit commerce a une condition plus modeste, et pour certains métiers dont l'exercice demande un faible capital (le bâtiment par exemple), la frontière avec les classes populaires est peu nette. D'une manière générale d'ailleurs, l'artisanat est plus proche du salariat agricole. Il s'y recrute en partie et une frange, dont l'importance est difficile à mesurer, y retourne. Par contre les passerelles entre artisanat et gros commerce sont beaucoup moins fréquentées.

L'ensemble de ces constatations autorise donc l'assimilation de l'artisanat beauceron à une petite bourgeoisie rurale proche des classes populaires. Son importance 
numérique montre qu'il serait très exagéré de simplifier à l'excès les structures sociales d'une région de grande culture comme l'est la Beauce : il y a, entre une classe dominante, mais très minoritaire, de grands exploitants agricoles, et la masse des ouvriers agricoles, une forte " classe moyenne "d'artisans, commerçants et petits cultivateurs (25). Son importance atténue les tensions sociales et explique pour une grande part la stabilité de la société rurale beauceronne au cours du XIXe siècle.

Jean-Claude FARCY

(C.N.R.S.)

\section{NOTES}

1. Faculté des Lettres et Sciences Humaines d'Aix-en-Provence. VIIIe Colloque d'Histoire sur l'artisanat et l'apprentissage. (Préface d'E. Labrousse), Aix-en-Provence, 1965, p.2.

2. H.G. Haupt, "La petite bourgeoisie, une classe inconnue 》 et $\mathrm{Ph}$. Vigier, "La petite bourgeoisie en Europe occidentale avant 1914 , in Le Mouvement social, juillet-septembre 1979, pp.3-20.

3. Les Archives Départementales d'Eure-et-Loir ont une série $P$ peu importante. Cette série n'est pas cotée.

4. Les Etats des montants des rôles généraux des contributions donnent pour chaque année et pour chaque commune d'Eure-et-Loir le nombre de cotes de patentes, le montant en principal des droits fixes et des droits proportionnels. La législation des patentes est compliquée et fluctuante. De manière simplifiée on peut dire que chaque patenté paie un droit fixe dont le montant varie selon la population de la commune d'exercice et selon l'importance de l'activité. Cette importance est déterminée par un classement de l'administration qui répartit les professions en plusieurs tableaux:

A : le commerce de gros, demi-gros et détail

B : le haut-commerce

C : l'industrie

$\mathrm{D}$ : les professions libérales.

Pour le tableau A qui seul nous intéressera, il y a 8 classes : A1 pour les marchands en gros; A2 pour les marchands en demi-gros... les classes A4 à A8 regroupant les petits commerçants et artisans. Au paiement du droit fixe s'ajoute celui d'un droit proportionnel qui est en rapport avec la valeur locative du local industriel muni de ses moyens de production, et de l'habitation du patentable. Les Registres de renseignements statistiques et administratifs des contrôles des contributions directes ont l'avantage d'indiquer par commune, mais pour une période limitée - de 1846 à 1879, et tous les trois ans -, le classement des artisans et commerçants, en indiquant en outre la valeur locative que représente chaque profession. Toutefois les métiers de même nature et situés dans la même classe sont regroupés : ainsi, dans telle commune, s'il y a 3 cabaretiers classés en $\mathrm{A} 6$, la valeur locative indiquée est celle des 3 cabarets pris ensemble.

5. Les listes de conscrits (Archives départementales d'Eure-et-Loir. Série R. non cotée) permettent une approche professionnelle des recrues, parfois de leurs parents, selon les années. En outre, pour le Premier Empire et les débuts du XXe siècle, ces listes indiquent les domiciles des parents et des recrues, ce qui permet d'aborder la question de l'exode rural.

6. Les listes nominatives des recensements $\rightarrow$ dont les Archives départementales d'Eure-et-Loir conservent une très belle collection, série $6 \mathrm{M}$ - ont l'avantage de donner la liste des habitants de chaque commune avec leur profession, tous les cinq ans, à partir de 1836 . On peut donc y relever la liste des artisans, selon leur activité, bien que le critère professionnel soit parfois très imprécis.

7. Les archives de l'Enregistrement (Archives départementales d'Eure-et-Loir. Série Q, non cotée) nous ont été utiles pour calculer le taux d'enrichissement des artisans en estimant, pour un échantillon donné, la fortune au mariage (Tableau des Contrats de mariages et Registres des Actes Civils Publics) et la fortune au décès (Tables alphabétiques des Successions et Absences et Registres de mutations par décès).

8. La Beauce d'Eure-et-Loir peut être assimilée aux deux arrondissements de Chartres et de Châteaudun, moins le canton de Brou qui relève du Perche. Pour l'analyse de l'artisanat proprement 
rural, nous avons naturellement éliminé de nos calculs les 3 cantons pour lesquels la population citadine est relativement élevée (Chartres-Nord, Chartres-Sud, Châteaudun).

9. On retrouve une place identique dans la Beauce du Loir-et-Cher, avec par exemple $23,2 \%$ dans le canton d'Ouzouer-le-Marché, d'après G. Dupeux, Aspects de l'histoire sociale et politique du Loir-et-Cher, 1848-1914, Mouton, Paris-La Haye, 1962, p.128.

10. Il est à remarquer que le nombre des patentés ne suit pas la même évolution dans toutes les régions de l'Eure-et-Loir. Dans le Perche (sud-ouest du département), la densité s'accroît de plus d'un cinquième entre 1851 et 1911 .

11. Par exemple, la loi du 2 juillet 1862 étant l'exemption déjà accordée en 1858 aux ouvriers travaillant chez eux ou chez les particuliers sans compagnon, apprenti, enseigne ni boutique, pour leur propre compte... comme à ceux qui travaillent à la journée ou à façon, aux ouvriers ayant enscigne ou boutique, s'ils ont les mêmes conditions d'exemption. 120000 personnes en bénéficieront dans toute la France. Des menuisiers, cordonniers, tonneliers, marchands de menues merceries, des bourreliers cessent ainsi de figurer dans les statistiques après 1862 . De 1862 à 1863 le nombre des droits fixes passe de 607 à 489 dans le canton de Janville, de 663 à 506 dans celui de Voves... Pour l'évolution de la législation, voir Jean Tardieu, Traité théorique et pratique des patentes, Paris, 1902.

12. Nous avons éliminé des calculs les 2 cantons « urbains 》 de Chartres Nord et Sud.

13. Cette constatation n'est pas contradictoire avec le fait que l'exode rural touche plus les artisans que les autres groupes sociaux. Ainsi nous pouvons mesurer - pour le début du XXe siècle le nombre de conscrits domiciliés en ville par rapport à leur origine rurale indiquée par le domicile des parents (les conscrits sont recensés généralement à leur lieu de naissance). Dans l'arrondissement de Chartres entre 1900 et 1904 , pour la partie rurale (ville de Chartres et chef-lieux de canton non compris) pour 100 conscrits d'origine rurale, un peu plus de 12 demeurent et travaillent en ville. Pour les artisans, le commerce alimentaire, et les autres secteurs du commerce, les chiffres sont respectivement de 13, 20 et 14. Dans les bourgs chef-lieux de canton c'est plus d'un jeune sur trois $(33,48 \%)$ qui est concerné par l'exode rural. Pour les 3 catégories de l'artisanat et du commerce que nous avons citées, les chiffres sont respectivement de $36,8,38,8$ et $42,8 \%$. Les enfants d'artisans et de commerçants prennent donc davantage le chemin de la ville. Les familles d'artisans semblent avoir plus d'enfants que les autres, en tout cas si l'on en juge par le recensement de 1851, et il est compréhensible que l'atelier ou la boutique qui ont besoin d'une main-d'œuvre très limitée et qui ne peuvent être continués que par l'un des enfants, beaucoup doivent quitter la campagne beauceronne.

14. M. Loriol, La France. Description géographique, statistique et topographique : L'Eure-etLoir par J. Doublet de Boisthibault, p.147.

15. Archives départementales d'Eure-et-Loir. Série P. Registre de renseignements statistiques du contrôle d'Auneau.

16. M. Barbier, meunier à Moutiers-en-Beauce (témoignage recueilli par le Groupe de Recherches sur les Traditions Populaires de Beauce), Le Vent du ch'min, Saint-Denis, 1979, pp.55-56. On notera que cela fait 19 "patentés 》 au début du XXe siècle pour une population de 400 habitants. Or il y a, d'après le Registre de renseignements statistiques du canton de Voves 24 patentés en 1846 pour une population de 510 habitants. On vérifie une fois de plus le maintien global de l'activité commerciale et artisanale dans la deuxième moitié du XIXe siècle. 1912.

17. Dr J. Bailhache, "La grande culture en Beauce 》, in La Science Sociale, juillet-décembre

18. Archives départementales d'Eure-et-Loir : II U, dossiers de la Cour d'Assises, lère session de 1857.

19. Idem, 4ème session de 1857 (témoignages datant de juin et de septembre 1852).

20. Archives départementales d'Eure-et-Loir : Registres de l'État-Civil de toutes les communes du canton de Chartres-Sud.

21. Archives départementales d'Eure-et-Loir : série $Q$, non cotée.

22. Archives départementales d'Eure-et-Loir : II U, dossiers de la Cour d'Assises, lère session de 1857.

23. Cette recherche se fait par l'intermédiaire des Tables alphabétiques de Successions et Absences qui relèvent tous les décès par canton et indiquent la référence à la déclaration de fortune consignée - quand elle existe - dans les Registres de mutations par décès. La difficulté d'accès aux archives de l'Enregistrement, les nombreux changements de domicile des personnes recherchées expliquent qu'on ne puisse retrouver la déclaration de succession de tous ceux qui se sont mariés dans le canton de Chartres-Sud entre 1811 et 1820 .

24. Pour la grande culture beauceronne, et pour une période similaire, le taux annuel d'enrichissement dépasse $7 \%$. 
25. La position de l'artisanat dans la société rurale comme les problèmes que pose son analyse sont développés par G. Gavignaud, "Aspects méthodologiques de l'artisanat rural en Roussillon au XIXe siècle ", in Bulletin du Centre d'histoire économique et sociale de la Région lyonnaise, $\mathrm{n}^{6} 3-4$, 1984 , pp.73-81. 